\title{
La domesticación de paisajes: ¿Cuáles son los componentes primarios del Formativo?
}

JOHN EDWARD STALLER ${ }^{1}$

\section{RESUMEN}

Al intentar definir los componentes básicos o primarios que caracterizan al Formativo en el oeste y el noroeste de Sudamérica, los arqueólogos se han encontrado con un creciente cuerpo de datos acerca de la transición hacia un modo de vida formativo mucho más variable de lo que se había pensado en un principio. Datos recientes procedentes de varias regiones de los Neotrópicos han demostrado que los rasgos primarios, a menudo adscritos a determinadas culturas, han variado ampliamente tanto en el tiempo como en el espacio, y generalmente están relacionados con factores locales, regionales, históricos y particularmente ecológicos. Las teorías anteriores que proponían una amplia dispersión de la revolución neolítica, hoy no están apoyadas por la información procedente del registro arqueológico. Esta información sugiere que el proceso de domesticación de especies vegetales puede ser enfocado y comprendido mejor a partir de una perspectiva ecológica y evolutiva más amplia, en términos de paisajes domesticados.

Palabras claves: Sudamérica-Formativo-domesticación vegetal.

\section{ABSTRACT}

In attempting to define the primary constituents that characterize a formative way of life in $W$ and $N W$ South America archaeologists have found that with ever increasing data the transition to a formative way of life is more variable than had ever been suspected. Recent data from various regions of the Neotropics has shown that the primary traits often ascribed to such cultures have been found to vary widely in time and space and are generally related to local, regional, historic, and particularly ecological factors. Previous theories of widespread Neolithic revolution are largely unsupported by the archaeological record, suggesting that the process of plant domestication is most efficiently discussed from a broader ecological and evolutionary perspective in terms of domesticated landscapes.

Key words: South America - Formative - vegetal domestication.

Recibido: septiembre 2005. Aceptado: febrero 2006.

1 Department of Anthropology, Research Associate, The Field Museum, Chicago, Illinois, ESTADOS UNIDOS. Email: jstaller@uky.edu

\section{Introducción}

La transición de la recolección a la producción de alimentos es un tema central para la investigación arqueológica que rodea al surgimiento y desarrollo de las civilizaciones. Sin embargo, a medida que se acumula la evidencia paleoetnobotánica, arqueológica y cronológica, parece haber una creciente carencia de consenso respecto a cuándo y dónde se debe trazar la línea divisoria en este continuum de desarrollo. En el siguiente análisis, introduciré una aproximación alternativa fuertemente enraizada en la teoría ecológica evolutiva, centrada en la reconstrucción de ecologías de subsistencia, en lo que mis colegas y yo hemos referido como "paisajes domésticos" (Terrell et al. 2003).

La presencia de maíz en sedimentos arqueológicos y basureros, o áreas de descarte de desechos alimenticios, ha sido, por lo general, citada como evidencia de lo que se consideran generalmente eventos significativos de un modo de vida formativo. Tal como se ha advertido en la comunidad arqueológica, recientes fechados directos por AMS de marlos tempranos procedentes de Mesoamérica, documentan un evento de domesticación más reciente en relación con la información previa. Los marlos más tempranos recuperados en Guilá Naquitz, Oaxaca, fueron datados en $\mathrm{C}^{14} 5410 \pm 40$ AP y $5420 \pm 40$ AP (Piperno y Flannery 2001: Tabla 1), mientras que en las cuevas de Tehuacán, México-Fase Coxcatlán-han arrojado fechas calibradas, de las cuales seis de ellos tienen fechas $\mathrm{C}^{14}$ comprendidas entre $3555 \pm 40 \mathrm{AC}$ y $2752 \pm 88$ AC. La datación promedio de los tres marlos más tempranos de la cueva Coxcatlán es de $2752 \pm 88$ AC (Benz y Long 2000: Tabla 1). En este caso, las dataciones de los marlos de Tehuacán, señalan dos mil años menos de antigüedad que la indicada para la Fase Coxcatlán, de acuerdo con las publicaciones originales (MacNeish 1985, 1994: 109-110, Tablas 3.2 y 3.4). Consecuentemente, algunos arqueólogos y paleoetnobotánicos han tenido que considerar una revisión de sus propuestas con respecto a los procesos y al tiempo asociado en relación a las transiciones agrícolas. 
Muchos estudiosos de la agricultura temprana consideran que algunas plantas, como el maíz, proporcionaron las bases económicas para el surgimiento de la civilización. Se destaca el rol del maíz (Zea mays L.) en la agricultura de subsistencia, relacionada con el sedentarismo y particularmente en la innovación precoz de la cerámica a lo largo y ancho de los Andes. Nuestro énfasis metodológico sobre los datos cronológicos vinculados con cultígenos de valor económico, que llevaron a la exclusión de otras plantas en el inventario paleoetnobotánico, indicaría en cierta medida que tales datos estaban sesgados, dando lugar a la reciente controversia que rodea los orígenes de Zea mays y consecuentemente la temprana dispersión del modo formativo de vida en las Américas. Más aún, esta aproximación ha obstaculizado, más que facilitado, el diálogo con los estudiosos de las ciencias biológicas y ecológicas, todo ello en detrimento de la interpretación arqueológica.

El desarrollo de las teorías y modelos neoevolutivos que hemos heredado; en otras palabras, nuestra búsqueda de transiciones agrícolas, centros de domesticación y revoluciones agrícolas, ha afectado profundamente nuestra comprensión del pasado. En el siguiente análisis, proporcionaré ejemplos de cómo tal forma de pensar ha modificado nuestra comprensión actual del desarrollo de un modo de vida "formativo" y por qué ha resultado no ser efectiva en proporcionar un consenso respecto de las importantes cuestiones de dicho desarrollo.

Hace casi un siglo, Herbert J. Spinden (1917) postuló la existencia de un estrato "formativo" que subyacía en las bases de la civilización en las Américas. Spinden, además, sugirió que los principales constituyentes de lo que él llamó "Cultura Arcaica" incluían agricultura del maíz, cerámica, figurillas antropomorfas, y la construcción de montículos ceremoniales. Kroeber (1930), por su parte, elaboró posteriormente un fundamento agrícola común para el Formativo con idénticas plantas alimenticias y similares técnicas textiles, metalúrgicas y arquitectónicas. Willey y Phillips (1958: 144) presentaron una interpretación histórico-desarrollista del Formativo definida: "por una presencia de agricultura de maíz y/o mandioca y la integración socioeconómica exitosa de tal agricultura dentro de una vida sedentaria bien establecida". Esta definición se ajustaba bien a las ideas de V. G. Childe, para el Neolítico del Viejo Mundo, en tanto se valora el cambio de una recolección de alimentos hacia la producción de los mismos. La evidencia arqueológica de América ha sido tradicionalmente analizada teniendo en cuenta si los datos se ajustaban a esta definición y, a pesar de alguna ambigüedad, la presencia y ausencia de tales constituyentes ha sido sistemáticamente aplicada como un esquema clasificatorio basado en estos criterios. Aproximadamente en la misma época en que esta definición aparecía impresa, MacNeish comenzó sus excavaciones en varias cuevas localizadas en el centro y norte de México, por un período de 20 años, con el objeto de desarrollar una secuencia cronológica que documentara los tipos de plantas que estaban siendo domesticadas (Mangelsdorf $e t$ al. 1967; MacNeish 1994). Ford, por otra parte, visualizó la dispersión del Formativo en términos de una difusión que comenzó aproximadamente hace unos 3000 años, con la aparición de la cerámica, implementos de molienda, figurinas, y agricultura de mandioca y maíz. Asimismo, Ford (1969: 5) percibió "centros de domesticación" y subdividió el Formativo en dos etapas: "Formativo Colonial" que comenzó aproximadamente hace 3000 años AC y el "Formativo teocrático", correspondiente al período posterior ( $c a .1200 \mathrm{AC}$ ).

Estos estudios pioneros estuvieron particularmente centrados en una innovación cerámica de carácter precoz. Esto se debe a que su presencia en el registro arqueológico era evidente y también porque se suponía que implicaba la temprana existencia de producción de alimentos. Sin embargo, a pesar de que existe suficiente evidencia paleoetnobotánica y arqueológica con respecto al cambio hacia la domesticación, todavía los arqueólogos no están de acuerdo en cuáles son los componentes primarios que caracterizan un modo de vida formativo. Varios análisis conducidos con método y diseñados específicamente para descubrir cuándo y dónde ocurrió el origen de la producción de alimentos, han generado conjuntos de datos que se concentran casi exclusivamente en las plantas con valor económico más tempranas (en particular maíz) con la exclusión de otras plantas silvestres en el inventario paleoetnobotánico. Los arqueólogos, al enfocar el proceso de domesticación exclusivamente en plantas económicas, han sesgado sus interpretaciones, puesto que han ignorado que esas plantas se modificaron por la selección consciente e inconsciente, y que inicialmente no cumplieron roles en términos de alimentos. Al considerar las consecuencias antes que las causas de la producción de alimentos, hubo una tendencia general en términos de suponer que los usos actuales de las plantas económicas reflejan 
necesariamente los roles de los especímenes del pasado. Centrándonos en una dinámica de sistemas, o dicho de manera más simple, en las causas antes que en las consecuencias de la "domesticación" de paisajes, podemos comenzar a preguntarnos sobre diferentes aspectos y hacer comparaciones que generen datos ecológicos y paleoetnobotánicos que nos permitan considerar variaciones subregionales con mayor detalle y reconsiderar por qué ciertos grupos de especies vegetales y animales son seleccionados en una región o área particular y no en otra (Terrell et al. 2003). Es posible que tal focalización pueda proporcionar una base para considerar cómo el consumo y/o la utilidad de ciertas especies han cambiado con el tiempo, por qué y cómo comenzaron a surgir los antiguos sistemas de agricultura intensiva. Deseo demostrar que los enfoques clasificatorios que hemos heredado, y nuestra perspectiva previa para la comprensión de los orígenes de la agricultura, no han sido exitosos en términos de proporcionar una base para documentar la existencia de un modo de vida formativo en estas variadas subregiones.
Precisamente, este trabajo incluye las siguientes áreas de estudio:

\section{La costa central de Perú}

Los arqueólogos que trabajan en los Andes advirtieron hace ya mucho tiempo que hay una significativa variación regional en cuanto a la explotación de plantas y animales. Aproximadamente 40 años atrás, Lanning (1967) informó acerca de una considerable variabilidad de lo que se estaba cultivando en los sitios del Período Inicial correspondiente a los valles del desierto costero de Perú. Sus observaciones se han visto luego apoyadas por recientes evidencias paleoetnobotánicas y arqueológicas procedentes de numerosos sitios localizados en los valles del norte y del centro del país.

Uno de estos sitios es Caral (Figura 1). La presencia de agricultura y construcción monumental ha sido, por lo general, considerada como indicador clave de la existencia de un modo de vida formativo. Las investigaciones realizadas por Shady y colaboradores

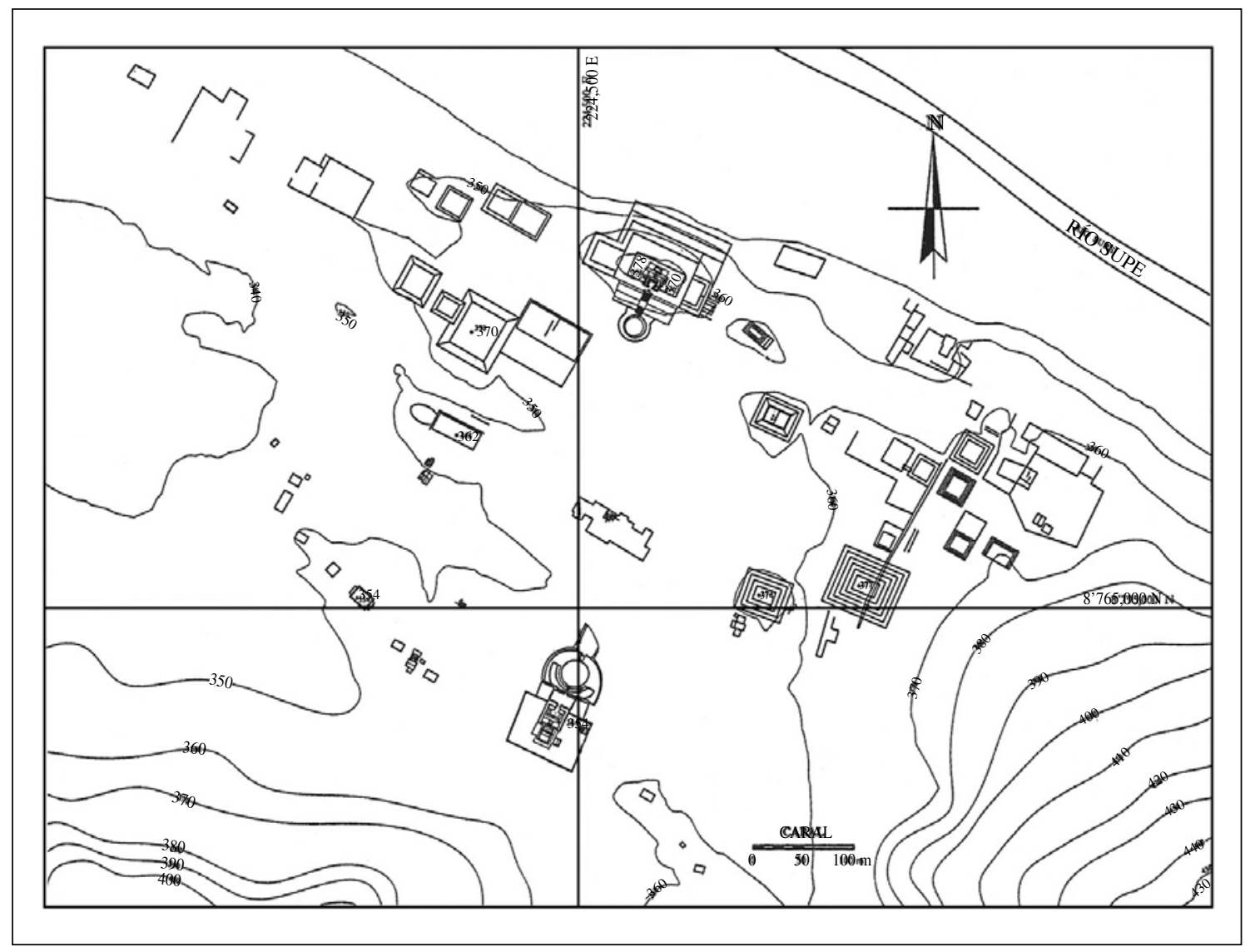

Figura 1. Plano de Caral en el valle de Supe, durante el Arcaico Tardío. Tomado de Shady y colaboradores (2003). 
a lo largo del valle de Supe, en la costa peruana, han documentado la presencia de sitios arcaicos tardíos con arquitectura monumental, tecnología de irrigación y evidencia de agricultura fechada entre 4090 y 3640 AP. El sitio de Caral es el más grande y más formalmente diseñado. Está localizado $23 \mathrm{~km}$ tierra adentro sobre una terraza fluvial de $25 \mathrm{~m}$, por encima de la planicie de anegamiento, conformado por una arquitectura monumental residencial y no residencial que cubre un área de aproximadamente 65 ha (Shady et al. 2003). A pesar de la escala de la construcción monumental y de la clara presencia de tecnología agrícola y de irrigación, estamos frente a una cultura perteneciente al Precerámico Tardío o Final. Este caso ejemplifica el hecho de que los cambios que condujeron a la dependencia de plantas domesticadas no implican necesariamente el control de la tecnología cerámica. Además, todos los cultígenos económicos consumidos en Caral son especies indígenas, algunas resultantes de procesos de domesticación y trasladadas desde distancias considerables y de distintos ambientes desérticos del valle costero de Supe. Los ejemplos incluyen maní (Arachis hypogaea), calabaza (Lagenaria siceraria), papa (Solanum tuberosum), achira (Canna edulis) y camote (Hipomoea batata).

Se acepta que la autoridad política centralizada impuso orden, control y coordinación en torno a las actividades económicas entre los habitantes del valle de Supe. El proceso sociocultural asociado a la complejidad y al desarrollo de una economía agrícola tuvo un carácter interdependiente y complementario con las sociedades costeras y las agrícolas del interior. En este sentido, las plantas económicas se intercambiaron localmente y fueron incrementadas bajo relaciones de interdependencia simbiótica, así como por una mayor división del trabajo.

Las prácticas de intercambio de recursos marítimos, como las anchoas secas por algodón (utilizado en telas y redes de pesca), así como otros cultivos, crearon un sistema cultural y económico combinado, lo cual benefició a ambos segmentos de la sociedad, promoviendo un sistema social jerárquico. Este proceso habría culminado con los inicios de un estilo de vida formativo determinado por la producción excedentaria. La ausencia de tecnología alfarera sugiere que no se le involucró entre las innovaciones esperadas, no siendo esencial para el desarrollo de las prácticas agrarias, para las instalaciones sedentarias ni en relación al procesamiento de múltiples cosechas de alimentos.

\section{El sur y suroeste de la costa ecuatoriana}

El sedentarismo y la innovación cerámica han sido observados durante mucho tiempo como fuertes indicadores de un estilo de vida formativo. Sin embargo, como ha sido tan claramente demostrado en varias regiones costeras de los Neotrópicos, ninguno de estos rasgos culturales puede ser considerado como claro indicador de una economía agrícola. La bien documentada Cultura Valdivia en la costa ecuatoriana es un caso muy representativo. A pesar de que la mayoría de los sitios Valdivia tempranos están localizados en la inmediata vecindad de la costa y la cerámica, las figurillas antropomorfas y la construcción de montículos ceremoniales datan de momentos muy tempranos ( $2700 \mathrm{AC}$ ). Sin embargo, el análisis de isótopos estables del carbón de los esqueletos tempranos de Valdivia indica que la dieta estuvo inicialmente centrada en plantas y animales terrestres, pero se torna cada vez más dependiente de los recursos marinos y acuáticos durante la parte final de la secuencia (Van der Merwe et al. 1993; Staller 1994, 2000; Tykot et al. 1996, 1996 Ms, 1996 Ms-a; Tykot y Staller 2002).

Aunque existían ciertas prácticas agrícolas, la adaptación Valdivia incorporaba una gama muy amplia de recursos de subsistencia (Van der Merwe et al. 1993; Tykot y Staller 2002). Las investigaciones regionales sobre asentamientos implican que las sociedades agrícolas completamente desarrolladas no aparecen hasta el final de la secuencia cultural de Valdivia, y aun entonces, las comunidades costeras continuaron estando especializadas en la explotación de los recursos acuáticos durante toda la secuencia prehispánica (Staller 2000).

En el caso de plantas económicas tales como el maíz y la mandioca, sobre la base de recientes datos radiocarbónicos, el primero no parece jugar un rol importante en la dieta de subsistencia, sino hasta la parte final de la secuencia, mientras que el rol de la segunda ha sido difícil de identificar directamente en forma arqueológica. Más aún, el maíz parece haber jugado inicialmente un rol importante en el ceremonial y ritualidad valdiviense, de modo que puede estar vinculado con el desarrollo temprano de interacciones a larga distancia y a la complejización sociocultural hacia el final de la secuencia cultural, pero no como un recurso económico (Staller y Thompson 2000, 2002; Staller 2003). Si bien para los Andes centrales se ha planteado una mayor dependencia a las plantas cultivadas, en los 
casos revisados la situación es algo similar pero con ciertas diferencias. El desarrollo de una economía agrícola se asocia en los sitios costeros de Valdivia con relaciones de interdependencia con los asentamientos del interior. Esto es evidente en la costa sur en la provincia El Oro, a juzgar por la presencia de conchas marinas y de la construcción de núcleos ceremoniales, tanto en el litoral como en el interior (Staller 1994, 2000). Estos sitios tienen en común el hecho de que se sitúan estratégicamente asociados a las rutas comerciales de larga distancia orientadas a las tierras altas (Hocquenghem 1993a, 1993b; Staller 2003). La extensión e integración innovadora de la cerámica durante la posterior Fase Chorrera, permitió vincular la cultura material directamente al desarrollo de sistemas sociales jerárquicos en el marco de una clara división social del trabajo, asociada a una economía basada en las nociones andinas tradicionales de reciprocidad y redistribución. Hay evidencia de prácticas de intercambio entre el sur de Ecuador y las sociedades norperuanas ya desde los 1500 AC (Hocquenghem 1991, 1993a, 1993b).

La evidencia de centros ceremoniales valdivienses ha sido documentada en varios sitios (Figura 2). Los sitios de Real Alto -El Encanto, Posora y Punta Arenas- indican una configuración circular o en forma de "U" rodeando una plaza central (Marcos 1988a y b). En el sector sur de la costa se excavó el centro ceremonial de La Emerenciana, documentándose un montículo ovalado en la parte noroeste del sitio (Figura 3) de 75 m N-S x 47 m E-O y aproximadamente $1.5 \mathrm{~m}$ de altura (Staller 1994: 319, 2001b: Figs. 10 y 14). En una terraza marina elevada en el sitio de San Lorenzo del Mate cerca

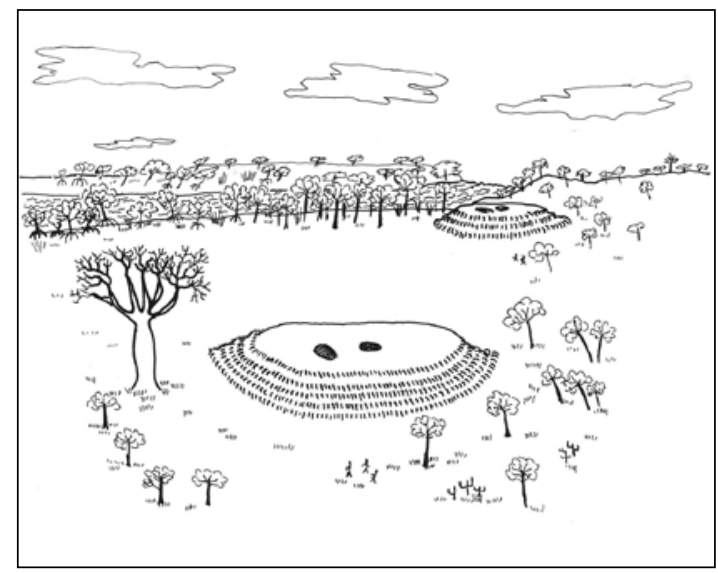

Figura 3. Reconstrucción de las plataformas ovales en La Emerenciana, Ecuador. Dibujo del autor.

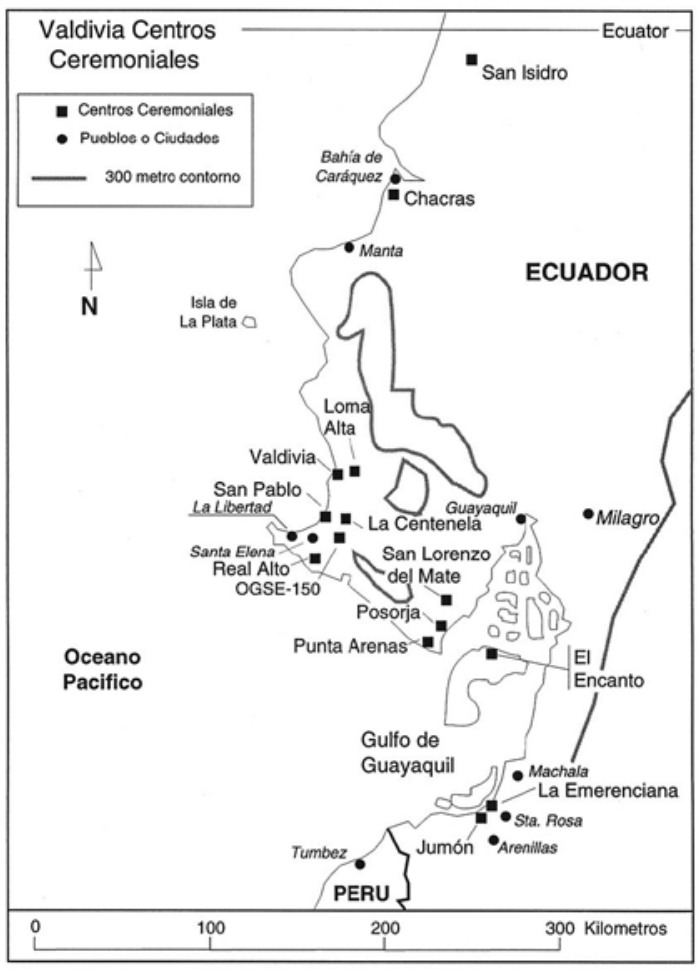

Figura 2. Centros ceremoniales de la Fase Valdivia, suroeste y sur de Ecuador. Dibujo del autor.

del río Mate, en la costa norte (San Isidro), al lado del río Jama, fue identificado un montículo artificial de $8 \mathrm{~m}$ de altura y aproximadamente $70-80 \mathrm{~m}$ en la base (Zeidler 1984; Staller 1994, 2000, 2001a y b, 2003). Estos datos indican una considerable variación en la configuración y la construcción de montículos ceremoniales y también en la cerámica diagnóstica regional para la porción final de la secuencia cultural de Valdivia (Staller 2000/2002). Estas pautas se contradicen fuertemente con los horizontes culturales de los Andes Centrales en cuanto posteriormente se asocian con los materiales relacionados con la extensión de la Fase Chorrera por la costa y las tierras altas, incluyendo las llamadas tradiciones de alfarería roja y blanca, propias de las tierras altas meridionales de Ecuador y el norte de Perú (Uhle 1920, 1922; Collier y Murra 1943; Jijon y Caamaño 1952; Bischof 1998; Tellenbach 1998).

\section{Noroeste de la costa colombiana}

En los estuarios costeros de la boca del río Magdalena y en la amplia planicie caribeña cercana a Cartagena, recientes investigaciones han ampliado las pros- 


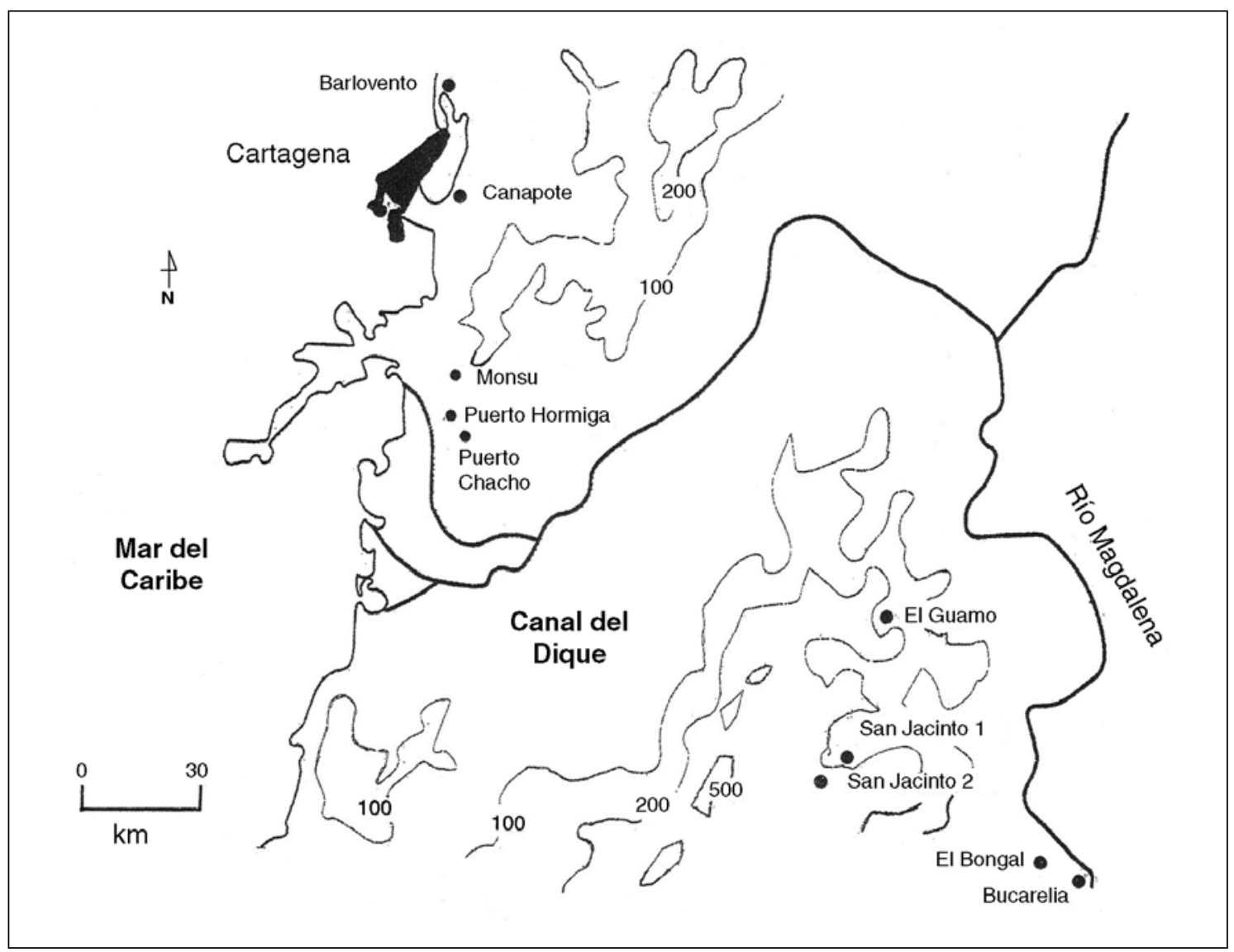

Figura 4. Sitios en el Canal del Dique, Colombia. Tomado de Raymond (1998: Fig. 2.8).

pecciones y excavaciones pioneras de Gerardo Reichel Dolmatoff y Alicia Dusan en los sitios de Puerto Hormiga, Monsú y San Jacinto (Figura 4). Estos y otros sitios en los alrededores de la región representan sólo algunos de los registros cerámicos más tempranos de América (Reichel-Dolmatoff 1955, 1957, 1961, 1965, 1985; Reichel-Dolmatoff y Dussán de Reichel 1955, 1961, 1962; Raymond et al. 1994). Sin embargo, en vez de documentar el desarrollo precoz de un modo de vida formativo, recientes prospecciones y excavaciones regionales en estos sitios y en el cercano de Puerto Chacho, han demostrado que se trata de cazadores recolectores marinos (Raymond 1998: 16). Los conchales en Puerto Hormiga y Puerto Chacho fueron ocupados durante un período de elevación del nivel del mar, mientras que otros sitios más tardíos, tales como Monsú, estuvieron ocupados cuando el nivel del mar era más bajo y la explotación de recursos se basaba en aquellos ambientes acuáticos fluviales más que en los marítimos, sugerido por la ausencia total de conchales (Reichel-Dolmatoff 1985; OyuelaCaycedo 1995). Precisamente, Oyuela-Caycedo
(1995) sostiene que la base costera rica en recursos facilitó el desarrollo de tecnologías de pesca y que esa disponibilidad de recursos no condujo a una adaptación hortícola ni a ocupaciones de largo plazo en localidades únicas. Los datos cronológicos indican que la cerámica estuvo asociada con ocupaciones estacionales, campamentos base y campamentos con propósitos especiales, casi un milenio antes de que los primeros montículos de valvas de moluscos aparecieran en esta región (Raymond 1998:17; Oyuela-Caycedo 1995: Fig. 3.1).

Aunque se trata de un campamento base de carácter estacional, Puerto Hormiga presenta una configuración circular anillada con un diámetro que oscila entre 16-25 m con depósitos domésticos que llegan hasta una profundidad de $1.2 \mathrm{~m}$ y que encierra un predio central o plaza libre de desechos ocupacionales (Figura 5). El sitio Barlovento, cronológicamente más tardío (1560-1030 AC), tiene una configuración circular similar constituida por la acumulación de conchas que conforman seis montículos casi simétricos, más o menos equidistantes, conformando 


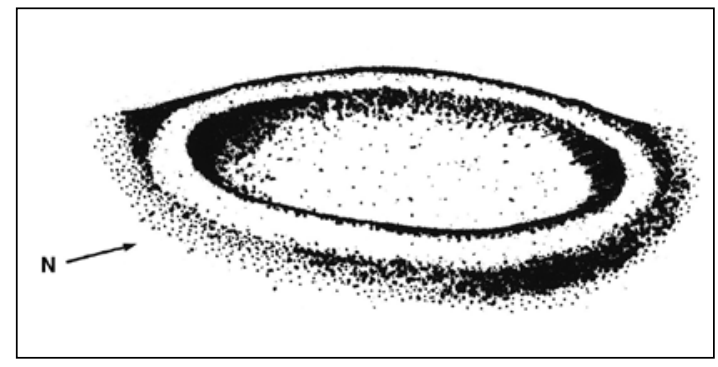

Figura 5. Montículo artificial en el sitio de Puerto Hormiga, Colombia. Tomado de Ford (1969).

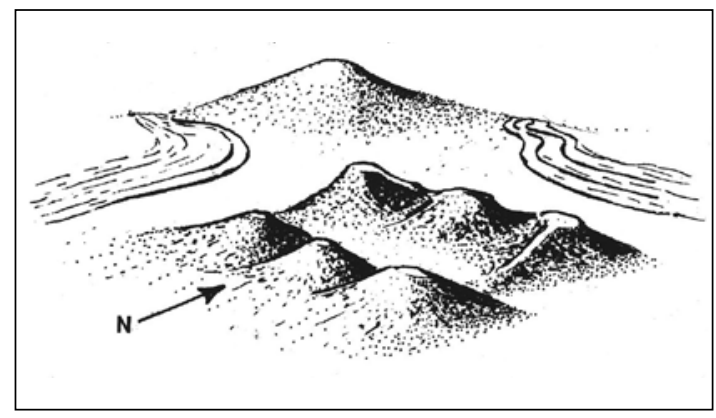

Figura 6. Montículo artificial en el sitio de Barlovento, Colombia. Tomado de Ford (1969: Carta 2-16).

un círculo que los conecta por sus bases (ReichelDolmatoff 1955: 247-272). Estos montículos cubren un área de 100 x 100 m y encierran una plaza central que mide 15 x 20 m (Figura 6).

La Serranía de San Jacinto presenta un ambiente transicional entre bosque y sabana, con una variación marcada en la precipitación estacional (Raymond 1998: 17). La más temprana evidencia de sedentarismo registrada en San Jacinto 2 coincide con la aparición temprana de conchales a lo largo de la costa. La baja densidad de fragmentos cerámicos en varios de estos sitios sugiere que la cerámica puede haber estado más involucrada en intercambios socioeconómicos y, a la vez, haber sido un elemento de estatus, antes que utilitario. Las elaboradas decoraciones de algunos fragmentos refuerzan esta idea (Oyuela-Caycedo 1995; Raymond et al. 1994; Raymond 1998). Los ricos recursos estuarinos parecen haber proporcionado un impulso hacia una mayor especialización así como también la disponibilidad estacional de plantas y animales terrestres, lo que habría favorecido la flexibilidad más que la especialización e intensificación de cultígenos específicos. Otro patrón interesante, aunque rara vez mencionado, es el hecho de que la innovación cerámica no se dispersó a las regiones aledañas de Puerto Hormiga y San Jacinto 1 y 2 , en algunos casos a lo largo de dos milenios, apoyando la idea de que la tecnología cerámica no estuvo necesariamente asociada con la dispersión regional de un modo de vida formativo. Los restos macrobotánicos y varios rasgos arqueológicos indican que las plantas silvestres, en especial las gramíneas, eran cosechadas estacionalmente (Raymond 1998: 17).

\section{Tierras Bajas de Panamá Central}

Otra región intensivamente estudiada respecto al cambio de la producción de alimentos es la costa pacífica de Panamá Central, a lo largo de los estuarios costeros del río Santa María. Los conchales de Cerro Mangote y Monagrillo, con ocupaciones tempranas que datan respectivamente del quinto y tercer milenio, son particularmente importantes (Cooke 1984, 1995; Cooke y Ranere 1984, 1992a, 1992b; Cooke et al. 1996). Esta región y estos sitios en particular han sido de importancia para los arqueólogos que estudian los cambios en el paisaje en tanto estos se relacionan con la transición entre la recolección y la producción de alimentos (Figura 7). Los sedimentos lacustres indican la presencia de quemas que se producen aproximadamente hacia los $5000 \mathrm{AC}$, además de una marcada declinación de las taxas forestales y un incremento en las gramíneas silvestres aproximadamente hacia los 2000 AC. Paralelamente en Monagrillo tiene lugar la aparición de cerámica, donde se incrementa el tamaño de los sitios y hay cambios en las estrategias de pesca (Cooke 1995; Cooke et al. 1996). Debe considerarse que hasta casi un milenio más tarde no aparecen villas o caseríos completamente sedentarios (Figura 8).

El patrón adaptativo de larga duración en la Bahía de Parita en Panamá Central, como el de la costa ecuatoriana, sugieren una flexibilidad adaptativa con respecto a la explotación de una amplia variedad de recursos acuáticos y terrestres. Sin embargo, el sedentarismo tiene lugar relativamente tarde, sugiriendo que la transformación de los patrones de subsistencia $\mathrm{y}$ asentamiento permanecieron independientes en relación con la presencia de especies domésticas particulares como la mandioca y el maíz. El conchal de Monagrillo está formado por un conjunto de crestas paralelas que miden alrededor de 100 x $80 \mathrm{~m}$ y corren longitudinalmente sobre una baja elevación natural, rodeando un patio central. Sobre la base de las excavaciones de Willey y McGimsey (1954), se pudo establecer que se hallaba a una profundidad mucho mayor de lo que se observa en superficie. 


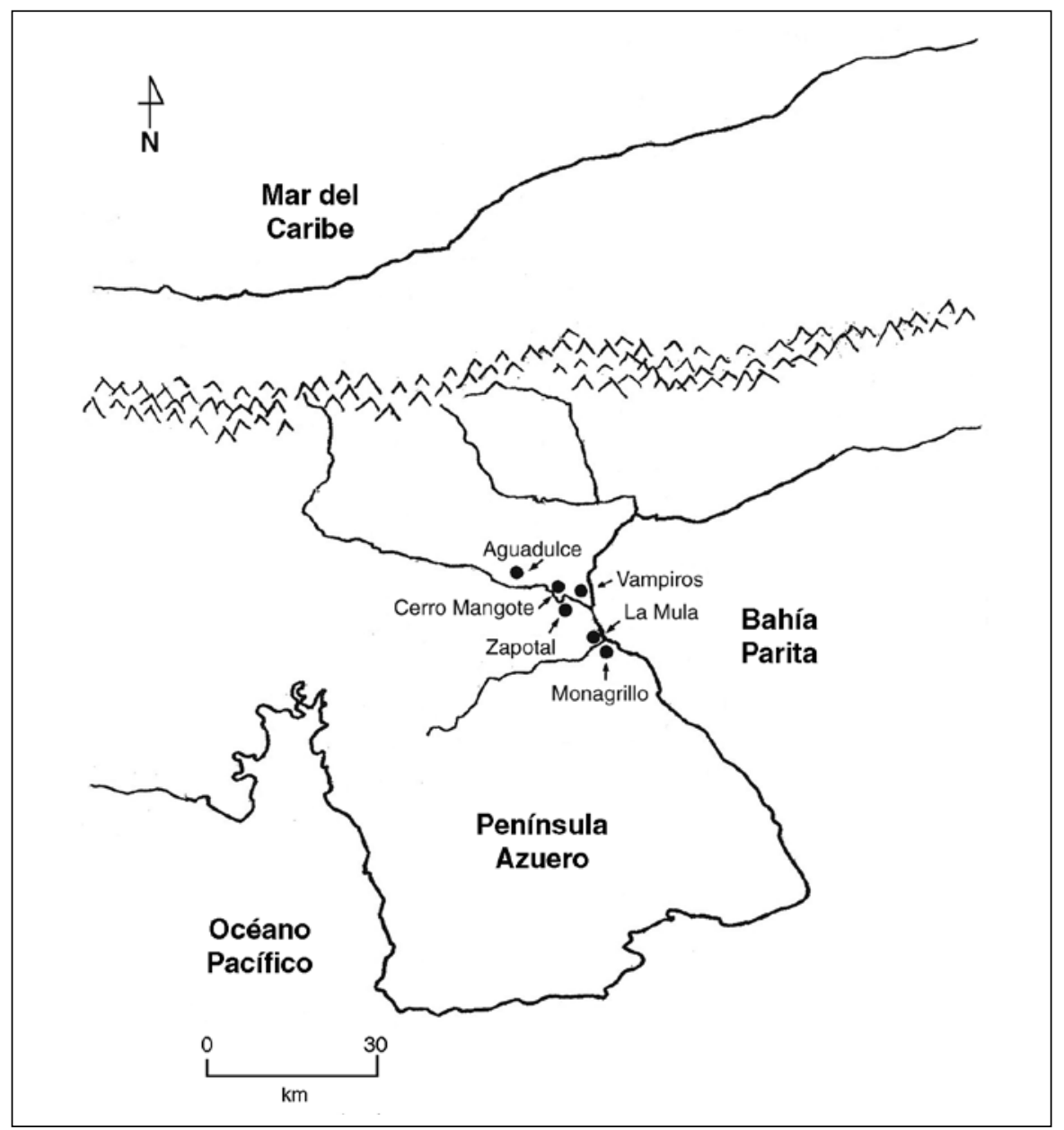

Figura 7. Asentamientos tempranos en la Bahía de Parita, Panamá. Tomado de Raymond (1998: Fig. 2.6).

Todavía no ha sido completamente determinado en qué medida estas estructuras representan una arquitectura ceremonial, aunque la configuración general del sitio es similar a la encontrada en otras regiones mencionadas en este análisis.

Estos datos indican que las clasificaciones basadas en los componentes primarios de un modo de vida formativo tampoco parecen aplicarse en esta región, como tampoco las caracterizaciones que consideran a estas poblaciones arcaicas como "hortícolas", "agrícolas" u "horticultores recolectores" (Piperno 1989: 548). Cooke (1992) enfatizó la explotación de larga duración de tubérculos silvestres, palmeras y otros árboles frutales como fundamentales para la subsistencia. Piperno (1989: 539) sugiere que la agricultura temprana representó una estrategia de bajo costo para amortiguar la escasez de recursos.
Sin embargo, esta interpretación carece de base intuitiva desde el punto de vista ecológico, ya que la respuesta adaptativa más común ante el riesgo producido por la escasez de recursos es un aumento del espectro dietario y una mayor flexibilidad adaptativa, antes que una especialización sobre recursos específicos. Cooke y Ranere (1992a y b) sugieren que el aumento poblacional fue el impulsor principal para la emergencia de un modo de vida formativo en Panamá Central, alrededor del 1000 AC, y su interpretación se apoya por un aumento en el tamaño y la distribución de los sitios. No obstante, el desarrollo de una agricultura intensiva en las planicies de anegamiento, con grandes ocupaciones permanentes focalizadas principalmente sobre el maíz, se cristaliza en esta región alrededor del 200 $\mathrm{AC}$ acompañado por estrategias especializadas en la explotación acuática. 


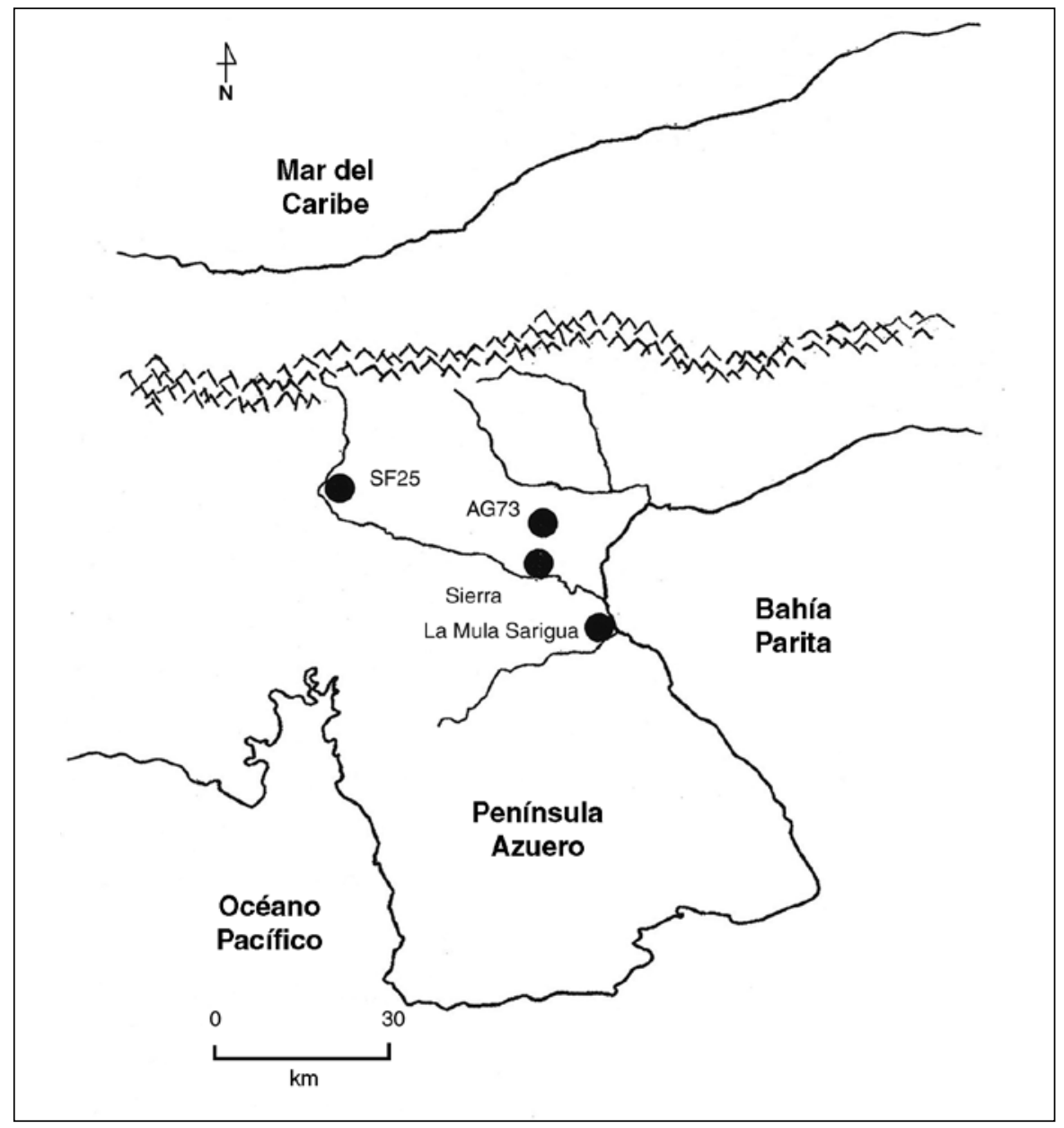

Figura 8. Asentamientos del 1000 AC en la Bahía de Parita, Panamá. Tomado de Raymond (1998: Fig. 2.7).

\section{Costa Rica septentrional}

El comienzo de un modo de vida agrícola en la región del Arenal del norte de Costa Rica se remonta a $c a$. 2000 AC (Hoopes 1987). Los pisos habitacionales domésticos enterrados en ceniza volcánica son considerados como evidencia de sedentarismo. Aunque el maíz puede haber estado presente en la región del Arenal tan temprano como por los 2000 AC, la evidencia más temprana documentada de maíz domesticado se remonta a alrededor del 700 AC (Horn y Kennedy 2001). Aunque el maíz estaba presente durante este período, los marcadores bioquímicos de los esqueletos asociados indican que fue una planta con valor secundario y no se convirtió en un cultígeno principal hasta los primeros siglos AC (Drennen 1996: 102). Varios investigadores sugieren que esto se debió a que el maíz se presenta en una forma relativamente "primitiva". Por otro lado, puede también indicar que no fue explotado o cultivado como una planta económica, sino que habría funcionado como un catalizador auxiliar para la fermentación, teniendo de este modo un significado social.

El rol social del maíz, desde su primer momento, coincide cronológicamente con la evidencia de producción especializada de cacao en los asentamientos del Formativo Temprano y Medio en la cercanía de la región central de Honduras (Joyce y Henderson 2001). En la región del Arenal, la evidencia de cultivos, sedentarismo y cerámica, aparece en asociación con un modo de vida formativo, pero como ya hemos visto, esta es la excepción antes que la regla en la mayor parte de los Neotrópicos.

La arquitectura ceremonial de este período está representada en el sitio Las Mercedes, constituido 
por montículos de tierra dispuestos formalmente (Willey 1971: 5-102). El montículo central en Las Mercedes es circular y tiene aproximadamente 6.5 $\mathrm{m}$ de alto, $30 \mathrm{~m}$ de diámetro y está cubierto por guijarros y cantos rodados fluviales (Figuras 9 y 10). Los montículos contiguos bajos y paralelos y las plataformas están dispuestos asimétricamente alrededor de un gran patio o plaza (Hartman 1901; Lothrop 1926; Mason 1945; Willey 1971). La construcción monumental incluye también tumbas elaboradas de forma rectangular $\mathrm{u}$ oval con paredes alineadas, pisos y techos sellados con trozos de piedra, con ofrendas que incluyen vasijas de cerámica, metates zoomorfos con forma de felino, las llamadas "piedras de altar", así como también adornos de oro (Willey 1971: 339-340). La escultura en piedra supuestamente asociada con este sitio sustenta aún más su función ceremonial (Figura 11).

En lugar de suponer una relación entre estos correlatos como indicadores de un modo de vida formativo, las estrategias de subsistencia deben ser documentadas y comprendidas en relación con conjuntos dinámicos de patrones ideológicos, ecológicos, socioeconómicos y sociopolíticos de comportamiento, en lugar de indagar hasta qué grado o en qué medida ellos se adecuan a un esquema de clasificación predeterminado (Terrell et al.2003). En vez de centrarse en las consecuencias de la domesticación, sugiero que las investigaciones futuras deberían adoptar un enfoque regional mucho más amplio en términos de paisajes domésticos. Es importante tener en cuenta que la focalización en el cambio hacia la producción de alimentos ha sesgado metodológicamente los datos microfósiles y conjuntos de datos sobre ciertos cultígenos específicos, y nos ha predispuesto a suponer que su rol económico en el presente puede ser directamente extrapolado para interpretar el pasado.

Tal como he documentado en un trabajo en colaboración con colegas de etnobotánica y de química de huesos, el maíz estuvo principalmente asociado con ceremonias rituales y fue consumido en la mayor parte de los Andes como un intoxicante fermentado y estrechamente asociado a rituales de intercambio (Tykot y Staller 2002; Staller 2003). Parece ser, tal como se visualiza en los datos que acabo de mencionar, que el rol temprano del maíz en estas regiones puede haber sido similar, particularmente cuando tanto la evidencia bioquímica como cronológica indican que recién se desarrolló como una reserva económica en los Neotrópicos aproximadamente y durante la misma época (500 AC-200 DC). Tal como Hoopes (1987) sugirió hace ya algún tiempo, debemos comenzar a alejar nuestra atención de los patrones "formativos" generalizados y sus supuestos correlatos asociados y empezar, en cambio, a focalizarla en la dinámica de estos procesos en una escala regional más restringida.

\section{Conclusiones}

Al considerar las consecuencias de la producción de alimentos, hubo una tendencia general a suponer que los usos actuales de las plantas económicas reflejan sus roles en el pasado (Terrell et al. 2003). Deberíamos comenzar a considerar la domesticación en términos de su manejo o conducción, tal como Flannery (1986) lo hizo en su análisis integrador sobre la domesticación en Guilá Naquitz. Fue David Rindos (1984) quien argumentó fuertemente contra la idea de "centros de domesticación", demostrando que la selección humana sobre el ambiente natural es una simbiosis microambiental y que la selección de ciertas plantas silvestres tiene un rango enorme de consecuencias sobre otras plantas y animales en la ecología.

Los antecedentes expuestos han llevado a que las investigaciones arqueológicas hayan enfocado la identificación de las más tempranas plantas domesticadas como evidencia de que el cambio agrícola se habría originado en ese período de tiempo. Las evidencias regionales aquí comentadas han demostrado que tales datos son una excepción más que una regla, tal como se ha observado en el caso de la región de Arenal (Costa Rica). En este sentido, la investigación de Flannery (1986) ha demostrado claramente que el Teosinte generalmente no se encuentra en los depósitos arqueológicos y que algunos pastos silvestres como Setaria jugaron un papel crítico en la subsistencia y adaptaciones arcaicas. Las investigaciones referidas, incluidas las cuevas de Tehuacan, indican que diferentes plantas fueron modificadas y de varias maneras, todas con seguridad de naturaleza fenotípicas o trasladadas desde sus ambientes naturales, modificándose genéticamente al ser adaptadas a condiciones diferentes, tanto ambientales como climáticas. Precisamente, tales pautas conductuales adquieren una importancia fundamental en la mejor comprensión de los procesos de domesticación conducentes a posteriori, a un estilo de vida formativo y a la economía agrícola. El énfasis persistente sobre la presencia de tempranas cosechas de plantas alimenticias, con exclusión de otras plantas del inventario macrobotánico, han 


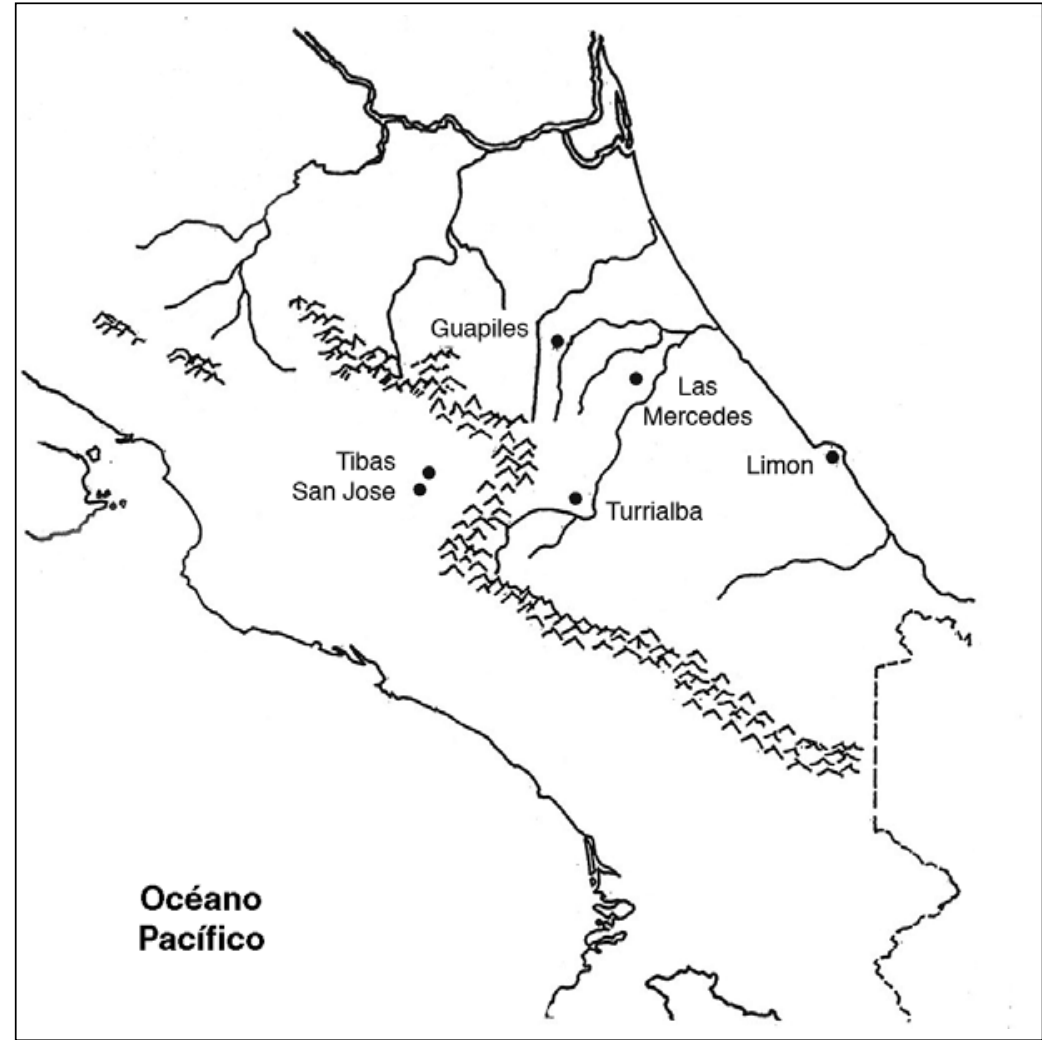

Figura 9. Asentamientos en la parte central de Costa Rica. Tomado de Snarkis (1981: Fig. 12).

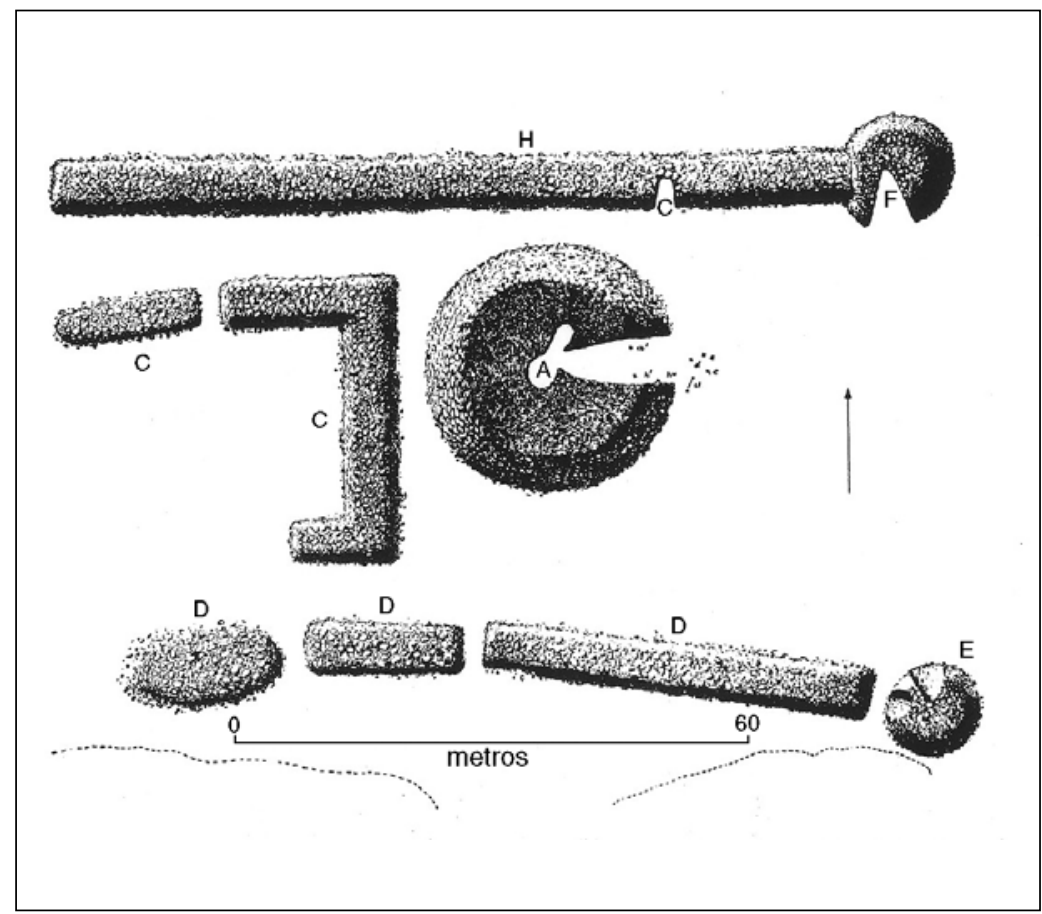

Figura 10. Plano del sitio de Las Mercedes, Costa Rica. Tomado de Willey (1971: Fig. 5-102). 


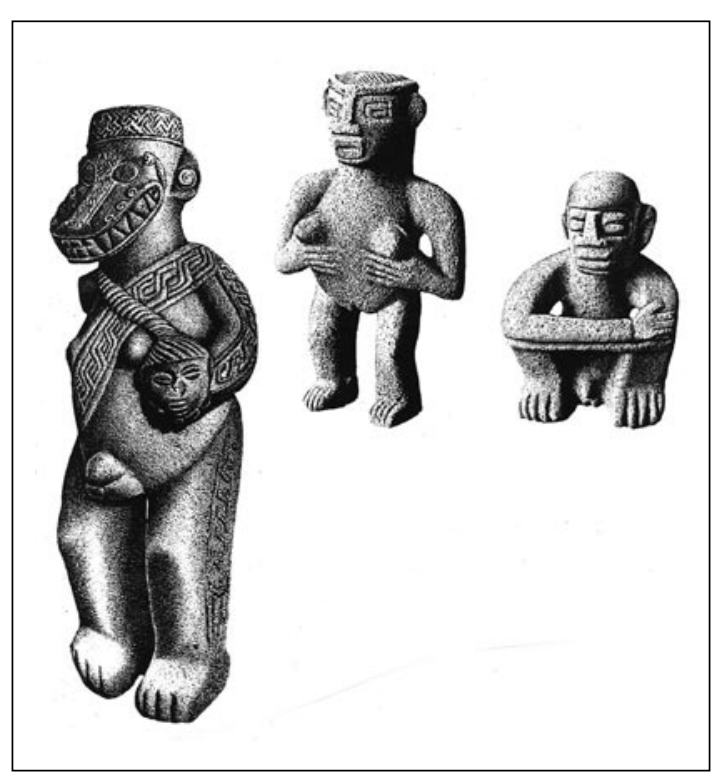

Figura 11. Esculturas en piedra de Las Mercedes, Costa Rica. Tomado de Willey (1971: Figs. 5-103 y 5-104).

predispuesto los planteamientos de interpretaciones basadas en la búsqueda de respuestas para confirmar preconceptos apriorísticos. Esta forma de hacer ciencia no es correcta, en cuanto ignora esencialmente los principios darwinianos de la selección natural y el papel de la adaptación humana en torno a la modificación del ambiente natural.

La domesticación de una especie por los grupos humanos depende de las características de la misma, de la cantidad y las partes de dicha especie que está(n) siendo seleccionada(s) (Terrell et al. 2003; véase también Smalley y Blake 2003). En el proceso de domesticación de una especie (conociendo cómo cosecharla), los usos de tal especie pueden cambiar sin tener en cuenta si dichos cambios asociados con la selección humana son intencionales o no (Terrell et al. 2003). En otras palabras, necesitamos comenzar a considerar no solamente qué especies están siendo cosechadas, sino también qué se está haciendo para cosecharlas. Al focalizarnos en las causas antes que en los comportamientos humanos involucrados en la "domesticación de paisajes", podemos comenzar a plantearnos diferentes preguntas y comparaciones que generen datos ecológicos y paleoetnobotánicos que nos permitirán considerar la variaciones subregionales con un mayor detalle $\mathrm{y}$, reconsiderar entonces por qué hay ciertos grupos de especies que están siendo seleccionadas en un área o región en particular. Estoy convencido de que tal focalización puede llegar a proporcionarnos una base para considerar cómo el consumo y/o la utilidad de ciertas especies ha cambiado a través del tiempo y el modo en que semejante variación influyó para que las sociedades arcaicas de América alcanzaran finalmente un modo de vida formativo.

Agradecimientos Mis agradecimientos más sinceros a mis colegas y amigos Osvaldo Mendonça y María A. Bordach (Universidad de Río Cuarto, Argentina) por sus aclaraciones sobre mi sintaxis y errores gramaticales, así como por su gran ayuda en la traducción final de este trabajo. Muchas gracias también al Sr. Olaf Jaime-Riveron (Universidad de Kentucky) por su ayuda en la traducción final del manuscrito. A pesar de la ayuda y consejo de estos eruditos, todas las interpretaciones y los datos presentados en este análisis fueron engendrados por el autor, quien toma toda la responsabilidad del contenido de este manuscrito.

\section{REFERENCIAS CITADAS}

BENZ, B. y A. LONG, 2000. Prehistoric maize evolution in Tehuacan Valley. Current Anthropology 41 (3): 459-465.

BISCHOF, H., 1998. Los orígenes de la civilización centroandina en la obra de Max Uhle. Indiana 15: 37-78.

COLLIER, D. y J. V. MURRA, 1943. Survey and excavations in Southern Ecuador. Field Museum of Natural History Publication 528, Chicago.

COOKE, R. G., 1984. Archeological research in Central, and Eastern Panama: A view of some problems. En The archaeology of Lower Central America, F. W. Lange y D. Z. Stone (Eds.), pp. 263-302. University of New Mexico Press y School of American Research, Albuquerque.
_ 1995. Monagrillo, Panama's first pottery (3800-1200 Cal. BC): Summary of research (1948-1993), with new interpretations of chronology, subsistence and cultural geography. En The emergence of pottery, W. K. Barnett y J. Hoopes (Eds.), pp. 169-184. Smithsonian Institution Press, Washington D. C.

COOKE, R. G. y A. J. RANERE, 1984. The Proyecto Santa María, a multidisciplinary analysis of prehistoric adaptations to a tropical watershed. En Recent developments in Isthmian archaeology: Advances in the prehistory of Lower Central America, F. Lange (Ed.), pp. 3-30. BAR International Series 212, Oxford.

1992a. Prehistoric human adaptations to the seasonally dry forests of Panama. World Archaeology 24 (1): 114-133. 
1992b. The origin of wealth, and the hierarchy in the central region of Panama (12000-2000 BP), with observations on its relevance to the history, and phylogeny of Chibchan-speaking polities in Panama, and elsewhere. En Wealth, and hierarchy in the Intermediate Area, F. W. Lange (Ed.), pp. 243-316. Dumbarton Oaks Research Library and Collection. Washington D. C.

COOKE, R. G., L. NORR, y D. R. PIPERNO, 1996. Native Americans, and the Panama landscape. En Case studies in environmental archaeology 15, E. J. Reitz, L. Newsom y S. Scudder (Eds.), pp. 103-125. Plenum Press, Nueva York.

DRENNEN, R. D., 1996. Betwixt and between in the Intermediate Area. Journal of Archaeological Research 4 (2): 95-131.

FLANNERY, K. V., 1986. Guilá Naquitz: Archaic foraging and early agriculture in Oaxaca. Academic Press, Nueva York.

FORD, J. A., 1969. A comparison of formative cultures in the Americas. En Contribution to Anthropology 11. Smithsonian Institution, Washington D. C.

HARTMAN, C. V., 1901. Archaeological researches in Costa Rica. Royal Ethnological Museum, Estocolmo.

HOCQUENGHEM, A. M., 1991. Frontera entre "Areas Culturales" nor y centroandinas en los valles y la costa del extremo norte peruano. Bulletin de l'Institut Français d'Etudes Andines 20 (2): 309-348.

- 1993a. Bases del intercambio entre las sociedades norperuanas y surecuatorianas: Una zona de transición entre 1500 AC y 600 DC. Bulletin de l'Institut Français d'Etudes Andines 22 (2): 443-466.

1993b. Rutas de entrada del mullu en el extremo norte del Perú. Bulletin de l'Institut Français d'Etudes Andines 22 (3): 701-719.

HOOPES, J. W., 1987. Early ceramics and the origins of village life in Lower Central America. Ph. D. Dissertation. Department of Anthropology, Harvard University, Cambridge, Massachusetts.

HORN, S. P. y L. M. KENNEDY, 2001. Pollen evidence of maize cultivation $2700 \mathrm{BP}$ at La Selva Biological Station, Costa Rica. Biotropica 29 (3): 191-196.

JIJON Y CAAMAÑO, J., 1952. Antropología prehispánica del Ecuador, Embajada de España Agencia Española de Cooperación Internacional, Museo Jacinto Jijón y Caamaño, Quito.

JOYCE, R. A. y J. S. HENDERSON, 2001. Beginnings of village life in Eastern Mesoamerica. Latin American Antiquity 12 (1): 5-23.

KROEBER, A. L., 1930. Cultural relations between North and South America. Proceedings of the $23^{\text {rd }}$ International Congress of Americanists (1928), pp. 5-22. Nueva York.
LANNING, E. P., 1967. Peru before the Incas. Englewood Cliffs, Prentice-Hall.

LOTHROP, S. K., 1926. Pottery of Costa Rica and Nicaragua. Contributions 8. Museum of the American Indian, Heye Foundation, Nueva York.

MACNEISH, R. S., 1985. The archaeological record on the problem of the domestication of corn. Maydica 30: 171-178.

1994. The origins of agriculture and settled life. University of Oklahoma Press, Norman y Londres.

MANGELSDORF, P. C., R. S. MACNEISH, y W. C. GALINAT, 1967. Prehistoric wild and cultivated maize. En The prehistory of the Tehuacán Valley, vol. 1: Environment and subsistence, D. S. Byers (Ed.), pp. 178-200. University of Texas Press, Austin.

MARCOS, J. G., 1988a. Real Alto: La historia de un centro ceremonial Valdivia. Biblioteca Ecuatoriana de Arqueología. Primera Parte ESPOL. Corporación Editora Nacional, Guayaquil.

1988b. Real Alto: La historia de un centro ceremonial Valdivia. Biblioteca Ecuatoriana de Arqueología. Segunda Parte ESPOL. Corporación Editora Nacional, Guayaquil.

MASON, J. A., 1945. Costa Rica stonework. Anthropological Papers 39 (3), American Museum of Natural History, Nueva York.

OYUELA-CAYCEDO, A., 1995. Rocks vs. clay: The evolution of pottery technology in the case of San Jacinto 1, Colombia. En The emergence of pottery, W. K. Barnett y J. W. Hoopes (Eds.), pp. 133-144. Smithsonian Institution, Washington D. C.

PIPERNO, D. R., 1989. Non-affluent foragers: Resource availability, seasonal shortages and the emergence of agriculture in Panamanian tropical forests. En Foraging and farming: The evolution of plant exploitation, D. R. Harris y G. C. Hillman (Eds.), pp. 538-554. Unwin Hyman, Londres.

RAYMOND, J. S., 1998. Beginnings of sedentism in the lowlands of Northwestern South America. En Recent advances in the archaeology of Northern Andes. In memory of ReichelDolmatoff, A. Oyuelo-Caycedo y J. S. Raymond (Eds.), pp. 10-20. The Institute of Archaeology, University of California, Los Angeles.

RAYMOND, J. S., A. OYUELA-CAYCEDO y P. CARMICHAEL, 1994. Una comparación de la cerámica temprana de Ecuador y Colombia. En Tecnología y organización de la producción cerámica prehispánica en los Andes, I. Shimada (Ed.), pp. 33-52. Pontificia Universidad Católica del Perú, Fondo Editorial, Lima.

REICHEL-DOLMATOFF, G., 1955. Excavaciones en los conchales de la costa de Barlovento. Revista Colombiana de Antropología 2 (2): 145-206.

1957. Momíl: A formative sequence in the Sinú valley, Colombia. American Antiquity 22: 226-234. 
1961. The agricultural basis of the sub-Andean chiefdoms of Colombia. En The evolution of horticultural systems in native South America: Causes and consequences, J. Wilbert (Ed.), pp. 83-89. Antropológica Suplemento 2. Sociedad de Ciencias Naturales La Salle, Caracas.

1965. Excavaciones arqueológicas en Puerto Hormiga (Departamento de Bolívar). Antropología 2, Ediciones de la Universidad de los Andes, Bogotá.

1985. Monsú: Un sitio arqueológico. Biblioteca Banco Popular, Textos Universitarios, Bogotá.

REICHEL-DOLMATOFF, G. y A. DUSSAN DE REICHEL, 1955. Investigaciones arqueológicas en la Sierra Nevada de Santa Marta, parte 4. Revista Colombiana de Antropología 5: 109-333.

_- 1961. Investigaciones arqueológicas en la costa pacífica de Colombia I: El sitio de Cupica. Revista Colombiana de Antropología. 10: 237-330.

- 1962. Investigaciones arqueológicas en la costa pacífica de Colombia II: Una secuencia cultural del bajo río San Juan. Revista Colombiana de Antropología 10: $237-330$.

RINDOS, D. R., 1984. The origins of agriculture: An evolutionary perspective. Academic Press, Orlando.

SHADY, R., C. DOLORIER, F. MONTESINOS y L. CASAS, 2003. Los orígenes de la civilización en el Perú: El área norcentral y el valle de Supe durante el Arcaico Tardío. En La ciudad sagrada de Caral-Supe. Los orígenes de la civilización andina y la formación del Estado prístino en el antiguo Perú, R. Shady y C. Leyva (Eds.), pp. 51-92. Instituto Nacional de Cultura, Lima.

SMALLEY, J. y M. BLAKE, 2003. Sweet beginnings: Stalk sugar and the domestication of maize. Current Anthropology 44 (5): 675-703.

SNARKIS, M. J., 1981. The archaeology of Costa Rica. En Between continents/between seas. The precolombian art of Costa Rica, E. Benson, (Ed.), pp. 15-84. Harry N. Abrams Publishers, Nueva York.

SPINDEN, H. J., 1917. The origin and distribution of agriculture in America. Proceedings of the $19^{\text {th }}$ International Congress of Americanists (1915), pp. 269-276. Washington D. C.

STALLER, J. E., 1994. Late Valdivia occupation in El Oro Province Ecuador: Excavations at the Early Formative Period (3500-1500 BC) site of La Emerenciana. Ph. D. Dissertation, Department of Anthropology, Southern Methodist University Dallas, Texas. UMI, Ann Arbor.

2000. Political and prehistoric frontiers: How history influences our understanding of the past. En The Entangled Past: Integrating History and Archaeology, M. Boyd, J. Erwin, y M. Hendrickson (Eds.), pp. 242-258. Proceedings of the $30^{\text {th }}$ Annual Chacmool Conference Calgary, Alberta (1997). The Archaeological Association of The University of Calgary, Alberta.
2000/2002. Explorando el contexto y el significado del intercambio a larga distancia en un paisaje sagrado: El caso del Formativo ecuatoriano. Cuadernos de Historia y Arqueología 54-56: 56-114.

2001a. Reassessing the chronological and developmental relationships of the Formative of coastal Ecuador. Journal of World Prehistory 15 (2): 193-255.

2001b. The Jelí Phase Complex at La Emerenciana, a Late Valdivia site in southern El Oro Province, Ecuador. Andean Past 6: 117-174.

2003. An examination of the paleobotanical and chronological evidence for an early introduction of maize (Zea mays L.) into South America: A response to Pearsall. Journal of Archaeological Science 30 (3): 273-280.

STALLER, J. E. y R. G. THOMPSON, 2000. Reconsiderando la introdución del maíz en el occidente de América del sur. Bulletin de l'Institut Français d'Etudes Andines 30 (1): 123-156.

2002. A multidisciplinary approach to understanding the initial introduction of maize into coastal Ecuador. Journal of Archaeological Science 29 (1): 33-50.

TELLENBACH, M., 1998. Acerca de las investigaciones de Max Uhle sobre las culturas tempranas de surecuador. Indiana 15: 269-353.

TERRELL, J. E., J. P. HART, S. BARUT, N. CELLINESE, A. CURET, T. DENHAM, C. KUSIMBA, K. LATINIS, R. OKA, J. PALKA, M. E. D. POHL, K. O. POPE, P. R. WILLIAMS, H. HAINES, y J. E. STALLER, 2003. Domesticated landscapes: The subsistence ecology of plant and animal domestication. Journal of Archaeological Method and Theory 10 (4): 323-367.

TYKOT, R. H. y J. E. STALLER, 2002. On the importance of early maize agriculture in coastal Ecuador: New data from the Late Valdivia Phase site of La Emerenciana. Current Anthropology 43 (4): 666-677.

TYKOT, R. H., N. VAN DER MERWE y N. HAMMOND, 1996. Stable isotope analysis of bone collagen and apatite in the reconstruction of human diet: A case study from Cuello, Belize. En Archaeological chemistry. Organic, inorganic and biochemical analysis, M. V. Orna (Ed.), pp. 355-365. ACS Symposium Series 625, American Chemical Society, Washington D. C.

TYKOT, R. H., N. VAN DER MERWE y S. ATHENS, 1996 Ms. The dietary significance of prehistoric maize in the northern Andes: An isotopic perspective. Ponencia presentada en The $61^{\text {st }}$ Annual Meeting of the Society for American Archaeology, New Orleans.

TYKOT, R. H., N. VAN DER MERWE y R. L. BURGER, 1996 Ms-a. Isotopic investigations of dietary dichotomies: The importance of maize and marine foods to Initial Period/Early Horizon subsistence in highland and coastal Peru. Ponencia presentada en International Symposium on Archaeometry, Urbana. 